\title{
Lithostratigraphie du Quaternaire de la vallée du Saint-Laurent : méthode, cadre conceptuel et séquences sédimentaires
}

\author{
The Quaternary Lithostratigraphy of the St. Lawrence Valley: \\ Method, Conceptual Framework and Sedimentary Sequences \\ Quaternär-Lithostratigraphie des Sankt Lorenz-Tals. \\ Methoden, begrifflicher Rahmen und Sedimentsequenzen
}

\section{Serge Occhietti}

Volume 44, numéro 2, 1990

URI : https://id.erudit.org/iderudit/032813ar

DOI : https://doi.org/10.7202/032813ar

Aller au sommaire du numéro

Éditeur(s)

Les Presses de l'Université de Montréal

ISSN

0705-7199 (imprimé)

1492-143X (numérique)

Découvrir la revue

Citer cet article

Occhietti, S. (1990). Lithostratigraphie du Quaternaire de la vallée du Saint-Laurent : méthode, cadre conceptuel et séquences sédimentaires. Géographie physique et Quaternaire, 44(2), 137-145.

https://doi.org/10.7202/032813ar

\section{Résumé de l'article}

Les dépôts du Pléistocène moyen et supérieur de la vallée du Saint-Laurent sont disposés selon une ordonnance caractéristique d'une vallée tectoniquement stable, périodiquement envahie par des glaces allochtones et inondée par des lacs glaciaires et des mers glacio-isostatiques. Chaque cycle climatique majeur est à l'origine d'une séquence sédimentaire composée de dépôts de type fluviatile, lacustre ou glaciolacustre, progression glaciaire, glaciaire, retrait glaciaire, glaciolacustre et marin. La base de chaque séquence est emboîtée par érosion fluviatile dans les séquences antérieures ou leurs témoins. L'érosion résulte du relèvement glacio-isostatique et de variations du niveau eustatique. Cette disposition générale explique: 1) l'homotaxie des unités laminées, des tills et des sédiments fluviatiles; 2) l'affleurement approximativement aux mêmes altitudes des faciès laminés et sableux d'âges différents; et 3) l'existence de buttes témoins d'unités plus anciennes enfouies sous les formations plus récentes. L'inventaire exhaustif des faciès, l'identification des unités par des critères intrinsèques et un inventaire palynologique systématique en cours ont permis: 1) de distinguer plusieurs types de rythmites indépendamment de leur position stratigraphique apparente (Varves de Deschaillons, Rythmites du Saint-Maurice et rythmites de Leclercville); 2) de découvrir une unité marine antérieure aux Sédiments de Saint-Pierre (Argile de La Pérade) et 3) de mettre en évidence des variations de la dynamique glaciaire pendant les phases d'englaciation et de déglaciation. D'après ces données et les données publiées, les dépôts quaternaires de la vallée peuvent être subdivisés en au moins trois séquences sédimentaires majeures avec englaciation, disposées entre l'Illinoien et le Wisconsinien supérieur, et d'au moins quatre unités fluviatiles et fluviolacustres représentatives de l'optimum climatique de l'Interglaciaire sangamonien, de deux épisodes intermédiaires et de l'Holocène. 


\section{LITHOSTRATIGRAPHIE DU QUATERNAIRE DE LA VALLÉE DU SAINT-LAURENT: MÉTHODE, CADRE CONCEPTUEL ET SÉQUENCES SÉDIMENTAIRES*}

Serge OCCHIETTI, Département de géographie et GÉOTOP, Université du Québec à Montréal, C.P. 8888, succursale A, Montréal, Québec H3C 3P8.

RÉSUMÉ Les dépôts du Pléistocène moyen et supérieur de la vallée du Saint-Laurent sont disposés selon une ordonnance caractéristique d'une vallée tectoniquement stable. périodiquement envahie par des glaces allochtones et inondée par des lacs glaciaires et des mers glacio-isostatiques. Chaque cycle climatique majeur est à l'origine d'une séquence sédimentaire composée de dépôts de type fluviatile, lacustre ou glaciolacustre, progression glaciaire, glaciaire, retrait giaciaire, glaciolacustre et marin. La base de chaque séquence est emboîtée par érosion fluviatile dans les séquences antérieures ou leurs témoins. L'érosion résulte du relèvement glacio-isostatique et de variations du niveau eustatique. Cette disposition générale explique: 1) l'homotaxie des unités laminées, des tills et des sédiments fluviatiles; 2) l'affleurement approximativement aux mêmes altitudes des faciès laminés et sableux d'âges différents; et 3) l'existence de buttes témoins d'unités plus anciennes enfouies sous les formations plus récentes. L'inventaire exhaustif des faciès, l'identification des unités par des critères intrinsèques et un inventaire palynologique systématique en cours ont permis: 1) de distinguer plusieurs types de rythmites indépendamment de leur position stratigraphique apparente (Varves de Deschaillons, Rythmites du Saint-Maurice et rythmites de Leclercville); 2) de découvrir une unité marine antérieure aux Sédiments de Saint-Pierre (Argile de La Pérade) et 3) de mettre en évidence des variations de la dynamique glaciaire pendant les phases d'englaciation et de déglaciation. D'après ces données et les données publiées, les dépôts quaternaires de la vallée peuvent être subdivisés en au moins trois séquences sédimentaires majeures avec englaciation, disposées entre l'Illinoien et le Wisconsinien supérieur, et d'au moins quatre unités fluviatiles et fluviolacustres représentatives de l'optimum climatique de l'Interglaciaire sangamonien, de deux épisodes intermédiaires et de l'Holocène.

* Contribution du $6^{\ominus}$ Congrès de l'Association québécoise pour l'étude du Quaternaire, sous la direction de Bernard Hétu

Manuscrit reçu le 14 novembre 1989; manuscrit révisé accepté le 27 novembre 1989
ABSTRACT The Quaternary lithostratigraphy of the St. Lawrence Valley: method, conceptual framework and sedimentary sequences. Since at least the Middle Pleistocene, the St. Lawrence Valley has been tectonically stable, periodically invaded by allochtonous ice, depressed by glacioisostasy, and flooded by glacial lakes and postglacial seas. The arrangement of the Quaternary deposits in the valley is related to this general setting of events. Sedimentary sequences composed of fluvial, lacustrine or glaciolacustrine, englacial, glacial, deglacial, glaciolacustrine and marine deposits are deposited during every main climatic cycle. The base of a sequence is inset into previous sequences, or their remnants, by cut and fill processes related to isostatic rebound and eustatic sea level changes. This general setting explains: 1) the homotaxy of the laminated units, of the tills and the sandy fluvial sediments; 2) the outcrops, at closely the same elevations, of laminated and sandy units of different ages; and 3) the outliers of some older units concealed by younger deposits. As a result of an exhaustive review of facies, of the identification of laminated units by intrinsic characters, and of a systematic and on going inventory of the pollen content, it has been possible: 1) to differentiate several rhythmites units (Deschaillons Varves, St. Maurice Rhythmites and Leclercville rhythmites) without using the apparent stratigraphic position; 2) to discover a marine unit older than the St. Pierre Sediments (La Pérade Clay); and 3) to put in evidence a complex ice dynamic during glacial invasion and ice retreat episodes. According to published and original data, the Quaternary deposits of the valley can be subdivided at least into three main sedimentary sequences of glacial units, laid down from the Illinoian to the Late Wisconsinan; and at least four fluvial and fresh water sequences deposited during the climatic optimum of the Sangamonian Interglaciation, two intermediate intervals, and the Holocene.

ZUSAMMENFASSUNG Quaternär-Lithostratigraphie des Sankt Lorenz-Tals. Methoden, begrifflicher Rahmen und Sedimentsequenzen. Die Ablagerungen des mittleren und höheren Pleistozän im Sankt Lorenz-Tal sind in einer Anordnung verteilt, die charakteristisch für ein tektonisch stabiles $\mathrm{Tal}$ ist, das periodisch durch allochtones Eis bedeckt und durch glaziale Seen und glazioisostatische Meere überschwemmt wurde. Jeder bedeutendere klimatische Zyklus führt zu einer Sediment-Sequenz, besthend aus Ablagerungen von Flüssen, Seen oder Glazioseen, glazialer Progression, Glazial, glazialem Rückzug, Glazioseen und Meer. Die Basis jeder Sequenz ist durch fluviatile Erosion in die Früheren Sequenzen oder ihre Zeugen eingefügt. Die Erosion hat ihren Ursprung in der glazio-isostatischen Anhebung und den Variationen des eustatischen Niveaus. Diese allgemeine Anordnung erklärt: 1) die Homotaxie der blättrigen Einheiten, der Tills und der fluviatilen Sedimente; 2) den Aufschluss der blättrigen und sandigen Fazies verschiedenen Alters in etwa gleicher Höhe; und 3) das Vorhandensein von Beleghügeln von gewissen älteren Einheiten, welche unter den jüngeren Formationen begraben sind. Ein erschöpfendes Inventar der Fazies, die Identifizierung der Einheiten durch innere Kriterien und ein systematisches palynologisches Inventar, das in Vorbereitung ist, haben gestattet 1) verschiedene Arten von Rythmiten zu unterscheiden, unabhängig von ihrer sichtbaren stratigraphischen Position (Warwen von Deschaillons, Rythmite von Saint-Maurice und Rythmite von Leclercville); 2) eine marine Einheit, die zeitlich vor den Sedimenten von Saint-Pierre (Ton von La Pérade) liegt zu entdecken und 3) Variationen in der glazialen Dynamik während der Vereisungs-und Enteisungsphasen deutlich zu machen. Diesen Daten und den publizierten Daten zufolge können die Quaternär-Ablagerungen des Tals in mindestens drei bedeutende Ablagerungssequenzen mit Vereisung unterteilt werden, welche zwischen dem Illinoium und dem späten Wisconsinium liegen, und in mindestens vier fluviatile und fluviolakustre Einheiten. 


\section{À LA MÉMOIRE DE ANDRÉ CAILLEUX, EN TÉMOIGNAGE DE SA TOLÉRANCE, DE SA GÉNÉROSITÉ, DE SA CURIOSITÉ SCIENTIFIQUE ET DE SON OUVERTURE D'ESPRIT}

\section{INTRODUCTION}

Le premier cadre stratigraphique cohérent du Pléistocène supérieur de la vallée moyenne du Saint-Laurent a été conçu entre 1955 et 1971 par Gadd $(1955,1960,1971)$, Karrow (1957) et Terasmae (1958). II comprenait deux unités glaciaires (Till de Bécancour et Till de Gentilly) précédées et suivies de varves et séparées par les Sédiments de Saint-Pierre de type fluviatile interstadiaire. Ce cadre résultait de la description des unités accessibles, selon la démarche analytique et l'état des connaissances de l'époque sur le Quaternaire. II représentait un progrès scientifique remarquable. Ce cadre est resté en vigueur jusqu'en 1984 (McDonald et Shilts, 1971; Gadd et al., 1972; Dreimanis et Karrow, 1972; Prest, 1975; MacDonald, 1971; Occhietti, 1982; Karrow, 1984; LaSalle, 1984). La découverte de dépôts fluviatiles recélant de la matière organique à Pointe-Fortune (Gadd et al., 1981; Veillette et Nixon, 1984) et à l'île aux Coudres (Brodeur et Allard, 1985) a étendu l'aire de distribution des dépôts de type Sédiments de Saint-Pierre, de la vallée de l'Outaouais jusqu'au moyen estuaire du SaintLaurent, sur une distance de $400 \mathrm{~km}$ (fig. 1). Le cadre stratigraphique de la vallée moyenne a été modifié par Lamothe $(1985,1987,1989)$ qui a démontré: a) que la séquence fluviatile-glaciolacustre-glaciaire exposée à Deschaillons cap Lévrard (fig. 1) était antérieure à la séquence Sédiments de Saint-Pierre - rythmites sus-jacentes - Till de Gentilly; et b) qu'un diamicton glaciaire est présent à la base des forages et par conséquent qu'il y a trois épisodes glaciaires représentés dans la vallée moyenne du Saint-Laurent. De plus, Anderson et al. (1988) ont rendu publique l'identification à Pointe-Fortune des témoins de l'optimum climatique du dernier Interglaciaire Sangamonien (stade isotopique 5e). Ils ont démontré que le till sous-jacent était par conséquent illinoien (ou antérieur), ce qui étend de façon irréfutable la colonne stratigraphique de l'ensemble de la vallée du Saint-Laurent au Pléistocène moyen.

Des travaux en équipe (Clet et al., 1986; Occhietti et al., 1989; et références ci-dessous), menés parallèlement à ces nouvelles recherches, ont mis en évidence : 1) la présence de nouveaux affleurements dans la vallée moyenne et dans l'estuaire du Saint-Laurent; 2) l'existence de nouvelles unités dans ces secteurs; 3) la nécessité de développer une approche adaptée à l'étude du Quaternaire de l'ensemble de la vallée du Saint-Laurent; 4) la nécessité de réanalyser et de réinterpréter selon les critères contemporains plusieurs unités déjà décrites dans le passé; et 5) la nécessité de repenser le cadre théorique de la stratigraphie du Quaternaire de la vallée du Saint-Laurent, selon les connaissances actuelles et à l'échelle de toute la vallée. Ce texte présente l'approche développée au cours de ces travaux. II propose un cadre stratigraphique ouvert qui permette d'intégrer toutes les unités connues et qui les dispose selon une ordonnance qui reflète la séquence des paléo-environnements de la vallée du Saint-Laurent. Rédigé à la suggestion de J.-S. Vincent, rédacteur en chef de Géographie physique et Quaternaire, le texte peut être considéré comme une introduction aux quatre articles de données stratigraphiques qui suivent: Bernier et Occhietti, 1990, Besré et Occhietti, 1990; Ferland et Occhietti, 1990a et b.

\section{MÉTHODE}

Au cours des travaux menés en collaboration depuis 1982 , nous avons constaté des contradictions entre les données publiées et les faits. Plus particulièrement, 1) de nombreux faits de terrain étaient passés sous silence, 2) les corrélations stratigraphiques étaient établies entre des types de faciès et non entre des unités définies par des critères caractéristiques et 3) les cadres lithostratigraphiques étaient focalisés sur les unités les plus apparentes et n'intégraient pas tous les faciès observés. Compte tenu de ces contradictions et de la très grande variété de faciès et d'unités observés, une méthodologie a été appliquée et adaptée au cours des travaux faits en équipe avec $M$. Clet (CNRS, Caen), F. Bernier, F. Besré et $P$. Ferland, étudiants à la maîtrise à l'Université du Québec à Montréal, plusieurs autres chercheurs et, récemment, avec J.-M. Lancery (Université du Québec à Trois-Rivières).

\section{1) INVENTAIRE EXHAUSTIF DES AFFLEUREMENTS}

Dans chaque secteur étudié, les affleurements ont été dégagés à la pelle, au jet d'eau sous pression ou avec une pelle mécanique. L'inventaire a été complété par des sondages à la tarière et quelques sondages sismiques réalisés par $\mathrm{J}$. Vézina et J.-P. Leroux du ministère des Transports du Québec. Le but de cet inventaire était d'observer, en continu, le plus possible de séquences locales, en insistant sur les contacts entre les unités et les parties masquées par les éboulis. L'inventaire a été accompli sans idée préconçue de la position stratigraphique, du nom, du rang ou de l'âge des unités observées. Tous les faciès ont été systématiquement levés, sans hiérarchie, quelles que soient leur épaisseur ou leur étendue latérale. Cette démarche s'est révélée fructueuse puisqu'elle a permis de découvrir notamment une nouvelle unité marine ancienne, épaisse seulement de 3,2 m (Ferland et Occhietti, 1990a), de nouveaux faciès glaciaires discontinus (Bernier et Occhietti, 1990) et de nouvelles lithozones dans les Sédiments de Saint-Pierre (Ferland et Occhietti, 1990b). L'inventaire exhaustif est un préalable à la reconstitution des paléoenvironnements et à la codification lithostratigraphique.

\section{2) DÉFINITION DES UNITÉS LITHOSTRATIGRAPHIQUES PAR DES CRITÈRES INTRINSĖQUES}

Dans la vallée du Saint-Laurent, il existe plusieurs unités de même faciès et d'âges différents qui se ressemblent et que les critères sédimentologiques classiques ne permettent pas de distinguer. De plus, ces faciès affleurent à des altitudes souvent identiques. En l'absence de méthodes de datation irréfutables, une grille d'analyse a été développée et adaptée aux types de dépôts observés dans la vallée. Cette grille d'analyse permet d'obtenir un faisceau de critères intrinsèques dont l'en- 


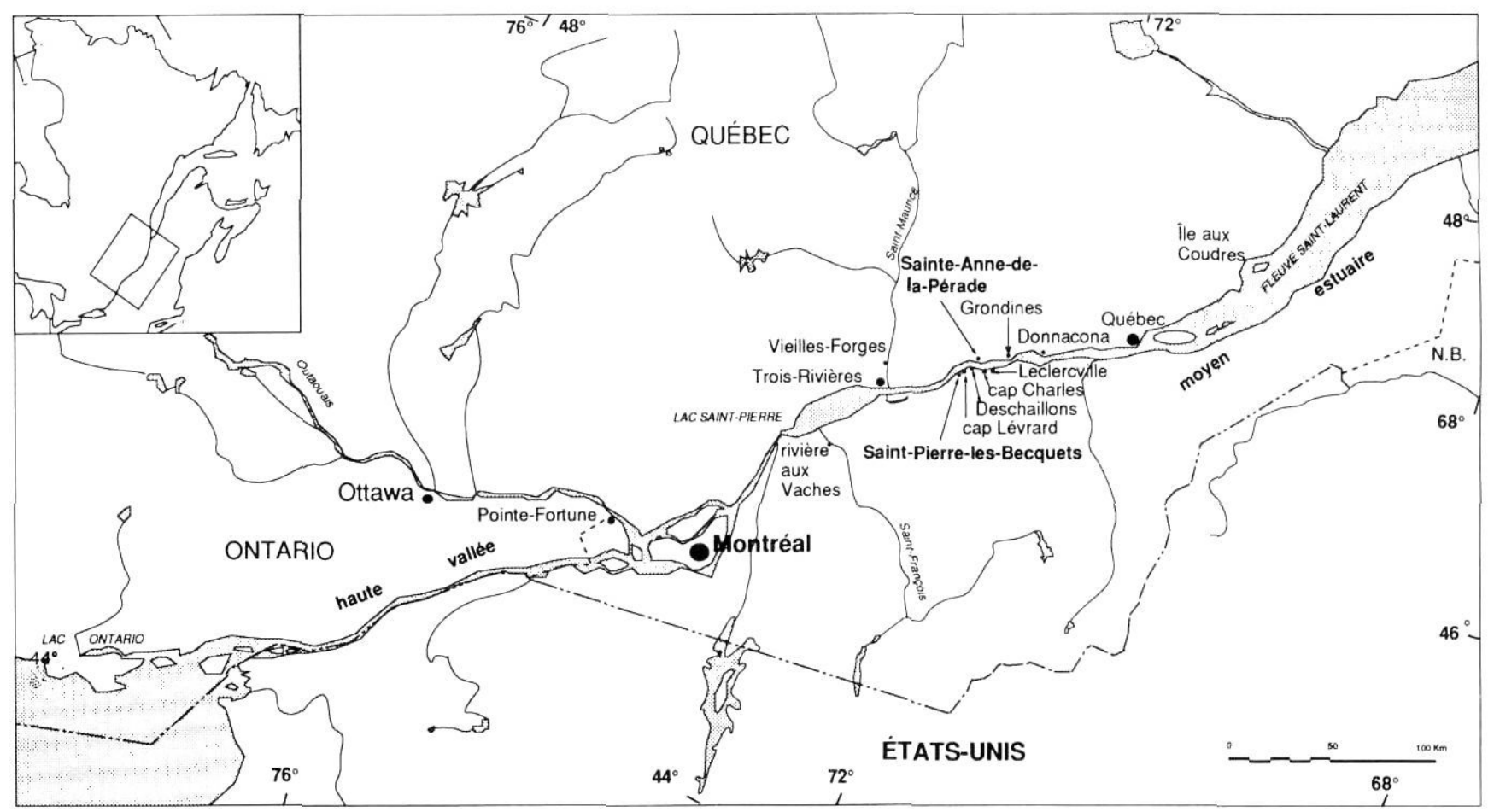

FIGURE 1. Carte de localisation des sites évoqués dans le texte.

Location map of the sites referred to in the text.

semble est caractéristique d'une unité. Une telle méthode a été utilisée par Sharpe et Barnett (1985), au sud de l'Ontario. Dans la vallée moyenne du Saint-Laurent, Besré et Occhietti (1990) contribuent à la distinction de trois unités laminées d'eau douce, dont les Varves de Deschaillons sensu stricto, en mesurant la variation verticale de l'épaisseur des doublets et des lits saisonniers et en tenant compte des bioturbations, du contenu pollinique et des microstructures sédimentaires. Les tills, définis longtemps avant que les classifications actuelles ne soient établies, sont actuellement très mal connus. Leur caractérisation selon les normes actuelles (cf. Parent, 1987) reste à faire. Hormis leur position stratigraphique et parfois leur contenu organique, il n'existe pas encore de critères intrinsèques pour distinguer les différentes unités fluviatiles.

\section{3) IDENTIFICATION DES SÉQUENCES LITHOSTRATIGRAPHIQUES}

La géométrie des unités, les discontinuités et les passages latéraux de faciès ont été établis en appliquant rigoureusement le travail d'inventaire en continu et l'analyse des critères intrinsèques. Cette démarche a permis de définir clairement la position stratigraphique relative des unités de plusieurs secteurs homogènes de la vallêe, notamment de Saint-Pierre-lesBecquets, Deschaillons - cap Charles, Sainte-Anne-de-laPerade - Grondines, Donnacona et de l'île aux Coudres. Quatorze unités distinctes ont été mises en évidence à SainteAnne-de-la-Pérade (Ferland et Occhietti, 1990a), dont deux tills antérieurs aux Sédiments de Saint-Pierre. D'autre part, le concept de lentilles glaciaires distinctes des nappes du till de Gentilly ou du Till de Bécancour telles que définies par Gadd (1971) commence à émerger des travaux de Lamothe (1989) et de Bernier et Occhietti (1990).

\section{4) INVENTAIRE BIOSTRATIGRAPHIQUE SYSTÉMATIQUE}

Les travaux d'Anderson et al. (1988) et de Clet et Occhietti (1988) montrent combien il est indispensable d'associer une biostratigraphie complète à la lithostratigraphie de la vallée du Saint-Laurent. L'inventaire biostratigraphique en cours par M. Clet, avec la collaboration de P. Richard (Laboratoire de paléoécologie et de palynologie, Université de Montréal) et de l'auteur, représente un outil stratigraphique très efficace. Par exemple, la concentration du pollen et le pourcentage d'arbres thermophiles permettent de distinguer les Varves de Deschaillons sensu stricto des rythmites postérieures aux Sédiments de Saint-Pierre (Besré et Occhietti, 1989) dont le contenu pollinique est abondant (jusqu'à 90000 grains $/ \mathrm{cm}^{3}$ ) et le pourcentage de grains d'arbres thermophiles moyen (jusqu'à $7 \%$ ) (Clet et Occhietti, 1988). D'autre part, le tamisage systématique à des fins micropaléontologiques a fait découvrir la présence de spicules d'éponges dans l'argile marine ancienne à Saint-Pierre-les-Becquets (Ferland et Occhietti, 1990a), ce que ne laissait pas prévoir la seule analyse sédimentologique.

\section{5) DATATION PAR LES MÉTHODES DISPONIBLES}

Actuellement, dans la vallée du Saint-Laurent, il existe peu de datations sur les unités plus anciennes que la limite de la méthode au ${ }^{14} \mathrm{C}$. L'âge des Sédiments de Saint-Pierre reste controversé. L'âge de 75 ka obtenu par enrichissement isotopique du ${ }^{14} \mathrm{C}$ sur du bois (Stuiver et al., 1978) est comparé à l'âge de $61 \pm 10$ ka calculé par thermoluminescence sur les sédiments (Lamothe et Huntley, 1988) et mis en relation avec l'âge de $80 \pm 10$ ka obtenu par la mesure du déséquilibre U/Th 
dans des concrétions des Varves de Deschaillons (HillaireMarcel et Causse, 1989). La mesure du taux de racémisation de l'acide aspartique sur du bois des Sédiments de Saint-Pierre (Occhietti et Rutter, 1982) donne des résultats actuellement difficiles à comparer. Ces limites de la géochronologie renforcent la nécessité d'établir une biostratigraphie et une lithostratigraphie aussi rigoureuses que possible.

\section{6) CORRÉLATIONS TRĖS RIGOUREUSES}

Afin d'éviter de retomber dans le piège des corrélations "sur papier" et faute de datations, la position relative des unités observées dans la vallée du Saint-Laurent est établie de façon extrêmement stricte. Deux unités mises en corrélation doivent nécessairement présenter les mêmes critères intrinsèques, le même type d'évolution biostratigraphique et sédimentologique, une position stratigraphique et une altitude compatibles avec le processus de mise en place qui leur est imputé. Cette démarche rigoureuse a permis d'éviter des erreurs d'interprétation stratigraphique, notamment d'amalgamer les deux tills inférieurs de Sainte-Anne-de-la-Pérade ou d'appeler Varves de Deschaillons toutes rythmites contenant des concrétions. Les variations latérales de faciès sont corrélées avec prudence. Conformément à cette démarche, les unités dont la superposition n'a pas été observée restent provisoirement en position stratigraphique ouverte. Ainsi, par exemple, la relation entre la séquence de Deschaillons - cap Lévrard (Lamothe, 1989) et de Sainte-Anne-de-la-Pérade (Ferland et Occhietti, 1990a) n'a pas été directement observée et reste donc en suspens.

Découlant de cette démarche, l'expérience semble montrer qu'il est préférable de définir des séquences de référence pour chaque secteur homogène de la vallée du Saint-Laurent plutôt que de tenter de corréler les unités sous une seule appellation valable pour toute la vallée. Cette remarque s'applique presque exclusivement aux unités antérieures aux Sédiments de Saint-Pierre.

\section{CADRE THÉORIQUE DE LA STRATIGRAPHIE DU QUATERNAIRE DE LA VALLÉE DU SAINT-LAURENT}

La reconstitution des nappes alluviales emboîtées de la vallée moyenne de la Seine par Lécolle (1984) et une recherche conjointe sur la superposition des cônes torrentiels et des nappes silteuses accomplie dans la vallée du Souss, au Maroc (Bhiry et al., sous presse), nous ont conduit à reconsidérer le cadre conceptuel de la stratigraphie de la vallée du SaintLaurent. II existe une disposition ordonnée des dépôts qui ne peut être correctement exprimée par une collection empirique d'unités. Plusieurs concepts explicites rendent compte de cette ordonnance.

\section{1) L'EMBOÎTEMENT DES UNITÉS FLUVIATILES}

Le concept explicite d'emboîtement des Sédiments de Saint-Pierre est formulé par Clet et Occhietti (1988) et généralisé par Ferland et Occhietti (1990b). Ce concept, appliqué habituellement aux vallées (Baulig, 1956), permet de concevoir l'ordonnance des unités de la vallée, non plus en une simple superposition d'unités avec des lacunes d'érosion, mais selon un système cohérent. Étant donné que la vallée du SaintLaurent semble tectoniquement stable depuis au moins le Pleistocène moyen, le niveau de base des unités fluviatiles sera le reflet de l'état de compensation glacio-isostatique et du niveau eustatique de chaque intervalle. En conséquence, il ne sera pas surprenant de trouver des unités fluviatiles et lacustres ou glaciolacustres, d'âges différents, à des altitudes sensiblement identiques. Les emboîtements sont également à l'origine de buttes témoins de dépôts plus anciens, dégagées par les chenaux fluviatiles et ultérieurement enfouies sous des dépôts plus récents. C'est le cas des buttes témoins de Deschaillons et de Sainte-Anne-de-la-Pérade - Grondines.

\section{2) LES SÉQUENCES SÉDIMENTAIRES}

Ce regroupement d'unités est classique. II a déjà été utilisé par Skinner (1973) dans la baie de James, par Miller et al.(1977) dans l'île de Baffin, par Vincent (1983) dans l'île de Banks et par Sharpe et Barnett (1985) dans le sud de l'Ontario. II s'applique parfaitement à la vallée du Saint-Laurent. Chaque cycle climatique majeur commence par une phase d'érosion fluviatile, suivie d'une accumulation fluviatile puis par des processus associés à la progression des glaciers, à l'invasion glaciaire, à la déglaciation et à l'invasion marine postglaciaire. Une séquence lithologique résulte de chaque cycle; bien que la séquence supérieure (tabl. I) puisse servir d'exemple, les séquences lithologiques antérieures ne sont pas nécessairement identiques. Chaque séquence est emboîtée dans les séquences précédentes ou leurs témoins. Les cycles climatiques courts, de 10000 ans et moins, et les fluctuations secondaires d'environnement, par exemple les épisodes froids sans englacement de la vallée et les fluctuations de la marge glaciaire, sont à l'origine d'unités ou de discontinuités intercalées entre les séquences ou comprises dans celles-ci.

\section{3) L'HOMOTAXIE DES UNITÉS}

L'idée de la répétition des mêmes faciès n'est pas nouvelle (Gadd, 1971; Lamothe, 1989). Toutefois, le concept explicite d'homotaxie, classique, reflète non seulement l'idée de répétition des mêmes séquences et des mêmes faciès d'âges différents mais également le risque d'erreur stratigraphique. La prise de conscience de l'homotaxie des unités laminées a conduit à distinguer ces unités par des critères indépendants de la position stratigraphique (Besré et Occhietti, 1990).

\section{4) LES VARIATIONS LATÉRALES DE FACIĖS}

Dans la vallée du Saint-Laurent, en dehors des phases d'englacement généralisé, des unités concomitantes mais de faciès distincts ont pu s'accumuler. Par exemple, l'épisode lacustre postérieur à la mise en place des Sédiments de Saint-Pierre présente des faciès proximaux aux tributaires du lac et un faciès distal au centre du lac (Besré et Occhietti, 1990). Sur le terrain, les silts laminés associés à ces faciès sont très différents et pourraient être considérés comme deux unités distinctes. Très peu d'études ont porté jusqu'à présent sur ces variations de faciès, à l'exception des sédiments de la Mer de Champlain. Une certaine difficulté de formalisation lithostratigraphique des unités est à prévoir entre l'axe et les marges de la vallée. 
TABLEAU I

Séquence sédimentaire postérieure à l'emboîtement des Sédiments de Saint-Pierre et sédiments fluviatiles holocènes, vallée moyenne du Saint-Laurent

\begin{tabular}{|c|c|c|}
\hline Environnements & Unités & Auteurs \\
\hline Fluviatile & sédiment du Saint-Laurent & Gadd, 1971 \\
\hline Lacustre d'exondation & silts du Lac Lampsilis & Elson, 1969 \\
\hline Marin & sédiments de la Mer de Champlain & Gadd, 1971 \\
\hline Glaciolacustre & varves à Candona & Parent, 1987 \\
\hline Retrait glaciaire & $\begin{array}{l}\text { faciès glaciomarins, fluvioglaciaires, de } \\
\text { contact glaciaire }\end{array}$ & $\begin{array}{r}\text { Gadd, } 1971 \\
\text { Occhietti, } 1982\end{array}$ \\
\hline Glaciaire & $\begin{array}{l}\text { Till de Gentilly } \\
\text { (comprend au moins deux lithozones) }\end{array}$ & $\begin{array}{r}\text { Gadd, } 1971 \\
\text { (Occhietti, 1977) }\end{array}$ \\
\hline Englaciation & turbidites de marge glaciaire & $\begin{array}{r}\text { Bernier et } \\
\text { Occhietti, } 1990\end{array}$ \\
\hline $\begin{array}{l}\text { Delta et épandage } \\
\text { lacustres ou glaciolacustres }\end{array}$ & Sables des Vieilles-Forges & Occhietti, 1982 \\
\hline Lacustre à sources multiples & Rythmites du Saint-Maurice & $\begin{array}{r}\text { Besré et } \\
\text { Occhietti, } 1990\end{array}$ \\
\hline Fluviatile & Sédiments de Saint-Pierre & $\begin{array}{r}\text { Gadd, } 1971 \\
\text { (révision de Ferland et } \\
\text { Occhietti, 1990b) }\end{array}$ \\
\hline
\end{tabular}

\section{5) LA FORTE DIVERSITÉ DE LA DYNAMIQUE GLACIAIRE}

À l'exception de la haute vallée du Saint-Laurent (MacClintock et Stewart, 1965; Clarke et Karrow, 1983), l'histoire glaciaire de la vallée du Saint-Laurent est très mal connue. Toutefois, ont sait maintenant que le mode de retrait glaciaire à la fin du Wisconsinien est assez complexe (Parent et Occhietti, 1988). Le complexe glaciaire du Cap Charles, dont la position stratigraphique est incertaine, indique des fluctuations d'une marge glaciaire au centre de la vallée (Bernier et Occhietti, 1990). Des cannelures d'âge non connu, observées pour la première fois à Grondines (fig. 1) par Lavoie (1987), sont la preuve, dans le haut estuaire du Saint-Laurent, d'un écoulement des glaces vers l'est. Dans le secteur de Donnacona, en aval de Grondines, les axes de la fabrique du till sous-jacent aux Sédiments de Saint-Pierre indiquent un écoulement glaciaire en compression vers l'ESE (Occhietti et al., 1989, fig. 18), tandis que des stries, découvertes sous un till apparemment antérieur au Till de Gentilly, indiquent un écoulement vers l'E-ESE. II est actuellement prématuré de dire s'il s'agit du même ou de plusieurs événements glaciaires distincts. Tous ces indices témoignent d'une régionalisation des événements glaciaires pendant les phases d'englacement et de déglaciation et de la nécessité d'une révision du cadre lithostratigraphique.

\section{6) LA FUGACITÉ DES ÉVÉNEMENTS}

Il existe une disproportion flagrante entre l'épaisseur de certaines unités et la durée de leur mise en place. Les rythmites de Leclerville, épaisses de plus de $25 \mathrm{~m}$, ne représentent que 300 ans de sédimentation (Besré et Occhietti, 1990). Au contraire, le Till de Gentilly, épais de quelques mètres, représente un laps de temps de l'ordre peut-être de 3000 à 60000 ans. Cette hétérogénéité des données continentales, bien connue, doit être clairement représentée dans les colonnes chronostratigraphiques. L'intérêt paléoenvironnemental des événements de courte durée demeure toutefois primordial.

\section{7) LA FORMALISATION DES UNITÉS LITHOSTRATIGRAPHIQUES}

Le cadre stratigraphique du Quaternaire de la vallée du Saint-Laurent est actuellement en cours de révision. La principale difficulté de cette révision concerne la réévaluation de la nature et de la position des unités. II faut à la fois définir de nouvelles unités, redéfinir d'anciennes unités et distinguer des unités antérieurement confondues (tabl. II). Conformément au code stratigraphique, il est souhaitable de décrire des séquences lithostratigraphiques de référence dans chaque secteur homogène de la vallée du Saint-Laurent. Dans un premier temps, afin de distinguer les principales unités homotaxiques (sables, sédiments laminés et tills), il semble nécessaire d'attribuer des noms formels aux principales d'entre elles. Par exemple, pour des raisons pratiques, il a été nécessaire de nommer les rythmites postérieures aux Sédiments de SaintPierre "Rythmites du Saint-Maurice" (Besré et Occhietti, 1990) pour les distinguer des Varves de Deschaillons plus anciennes (Lamothe, 1989). La révision des Sédiments de Saint-Pierre, unité pivot de toute la stratigraphie de la vallée, était devenue 
TABLEAU ॥

Réinterprétation d'unités lithostratigraphiques du Pléistocène, vallée moyenne du Saint-Laurent

\begin{tabular}{lc}
\hline $\begin{array}{l}\text { Anciennes } \\
\text { unités }\end{array}$ & $\begin{array}{c}\text { Nouvelles } \\
\text { unités } \\
\text { Unité }\end{array}$ \\
\hline
\end{tabular}

$\begin{array}{ll}\text { Sédiments de } & \text { Sédiments de } \\ \text { Saint-Pierre } & \text { Saint-Pierre }\end{array}$

(Gadd, 1971)

Varves de

Deschaillons

(Gadd, 1971;

Karrow, 1957:

Occhietti, 1982)

(critères intrinsèques définis par Besré et
Varves de Deschaillons (position stratigraphique) révisée par Lamothe, 1989) Occhietti, 1990)

Rythmites du SaintMaurice (position stratigraphique révisée par Lamothe, 1985) (critères intrinsèques définis par Besré et Occhietti, 1990)

rythmites de Leclercville (Besré et Occhietti, 1990)

varves de déglaciation sans nom (Ferland et Occhietti, 1990a)

varves du cap Lévrard, affleurent à marée basse ou sous un till rouge (Gadd, 1971) (nom informel proposé par Occhietti, 1982)
"Varves du Cap Lévrard" $=$ Varves de Deschaillons (Lamothe, 1989)
Position stratigraphique

Stratotype
Saint-Pierre-les-Becquets (Gadd, 1971) (révisé par Ferland et Occhietti, 1990b)

sous - "varves grises" (Lamothe, 1989) ou Rythmites du Saint-Maurice (Besré et Occhietti, 1990)

$$
\begin{gathered}
\text { sur - rythmites sans nom } \\
\text { (Lamothe, 1989) }
\end{gathered}
$$

sous - Varves de Deschaillons à la briqueterie (Lamothe, 1989)

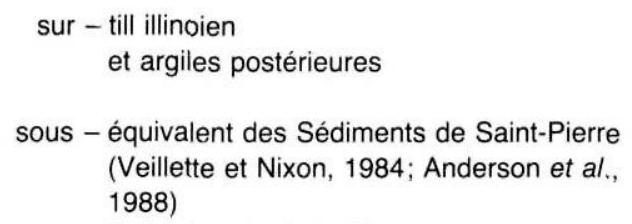
1988)
sur - Rythmites du Saint-Maurice définies par Besré et Occhietti (1990)
Les Vieilles-Forges
(Occhietti, 1982) et anciennement confondues avec les Varves de Deschaillons (Gadd, 1971; Occhietti, 1982)

sous - Till de Gentilly

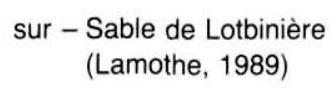

briqueterie de Deschaillons (Lamothe, 1989)

non formel, à Pointe-Fortune (Anderson et al.., 1988)

briqueterie de Deschaillons (Gadd, 1971)

(révisé par Lamothe, 1989)

Les Vieilles-Forges (Besré et Occhietti, 1990)

non formel, à Leclercville

non formel, à Sainte-Annede-la-Pérade (Ferland et Occhietti, 1990a)

coupe de référence au cap Lévrard (Lamothe, 1989), stratotype à la briqueterie de Deschaillons (Gadd, 1971; Lamothe, 1989) 


\begin{tabular}{|c|c|c|c|}
\hline \multirow[t]{2}{*}{$\begin{array}{l}\text { Anciennes } \\
\text { unités }\end{array}$} & \multicolumn{3}{|l|}{$\begin{array}{l}\text { Nouvelles } \\
\text { unités }\end{array}$} \\
\hline & Unité & Position stratigraphique & Stratotype \\
\hline & $\begin{array}{l}\text { rythmites de Leclercville } \\
\text { (Besré et Occhietti, 1990) }\end{array}$ & $\begin{array}{l}\text { sur - unité non connue } \\
\text { sous - complexe glaciaire du cap Charles } \\
\text { et Till de Gentilly } \\
\text { (Bernier et Occhietti, 1990) }\end{array}$ & $\begin{array}{l}\text { coupe de référence au cap } \\
\text { Charles (Bernier et Occhietti, } \\
\text { 1990), le stratotype non formel } \\
\text { est à Leclercville (Besré et } \\
\text { Occhietti, 1990) }\end{array}$ \\
\hline & rythmites sans nom & 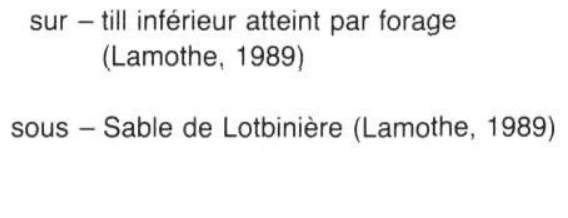 & $\begin{array}{l}\text { affleurements à marée basse en } \\
\text { aval de Deschaillons (Bernier et } \\
\text { Occhietti, 1990); probablement } \\
\text { équivalentes aux rythmites } \\
\text { atteintes par forage par Lamothe } \\
\text { (1989) }\end{array}$ \\
\hline
\end{tabular}

impérative (Clet et Occhietti, 1988; Ferland et Occhietti, 1990b). Toutefois, le rang formel (membre, formation) de ces unités peut rester en suspens, dans l'attente des analyses biostratigraphiques et d'un inventaire plus complet. Dans un deuxième temps, les corrélations lithostratigraphiques entre les secteurs de la vallée seront établies sur une base rigoureuse (critères intrinsèques, position stratigraphique relative et datation) et intégrées dans un cadre climatostratigraphique et chronostratigraphique d'ensemble.

\section{SÉQUENCES SÉDIMENTAIRES CONNUES DU QUATERNAIRE DE LA VALLÉE DU SAINT-LAURENT}

D'après les données déjà publiées ou présentées publiquement et les travaux publiés dans ce volume (Bernier et Occhietti, 1990; Besré et Occhietti, 1990; Ferland et Occhietti, 1990a et b), les séquences sédimentaires suivantes peuvent être identifiées:

1) séquence glaciaire de l'lllinoien (ou antérieure), représentée par le till inférieur de Pointe-Fortune (Anderson et al., 1988). Les termes de retrait glaciaire, d'inondation lacustre et marine sont peut-être représentés par les rythmites sous le niveau du fleuve et autres unités signalées par Simard (1971) et Lamothe (1989);

2) intervalle fluviatile de l'optimum climatique du Sangamonien représenté à Pointe-Fortune par des sables et silts à Carya (Anderson et al., 1988);

3) une séquence fluviatile et d'englaciation antérieure aux Sédiments de Saint-Pierre, identifiée par Lamothe (1989) au cap Lévrard-Deschaillons (Sable de Lotbinière, Varves de Deschaillons, Till de Lévrard);

4) plusieurs séquences locales de glaciation-déglaciation, antérieures aux Sédiments de Saint-Pierre et dont l'âge incertain peut varier entre la fin de l'lllinoien et la fin du Wisconsinien inférieur:

- séquence atteinte par forage sous le fleuve à Deschaillons et attribuée à l'Illinoien par Lamothe (1989);

- séquence de la rivière aux Vaches décrite par Gadd (1971), incluant un till inférieur (nommé till d'Odanak par Lamothe, 1987 ) et des varves de déglaciation (Gadd, 1971);
- séquence du till inférieur et des varves sus-jacentes de Sainte-Anne-de-la-Pérade (Occhietti et al., 1989; Ferland et Occhietti, 1990a);

- séquence du till intermédiaire, des faciès glaciolacustres et de l'Argile marine de La Pérade, à Sainte-Anne-de-la-Pérade, qui ne semble pas dater de la fin de l'lllinoien (Ferland et Occhietti, 1990a);

5) plusieurs séquences glaciaire-marge glaciaire et glaciolacustre-glaciaire, d'âge incertain:

- séquence de la haute vallée du Saint-Laurent: représentée par des varves, le "Till de Malone", des varves, un complexe glaciaire intermédiaire, des varves et un till supérieur ( "Till de Fort-Covington") (MacClintock et Stewart, 1965; Clark et Karrow, 1983);

- séquence de la région de Montréal jusqu'au piémont des Appalaches: Till de l'Ange-Gardien (Prichonnet, 1982), varves, Till de Saint-Jacques (LaSalle, 1984);

- séquence glaciaire-marge glaciaire-glaciaire sous les Sédiments de Saint-Pierre à Donnacona (Clet et al., 1986; Occhietti et al., 1989);

6) séquence postérieure à l'emboîtement des Sédiments de Saint-Pierre, très développée dans la vallée moyenne du SaintLaurent (tabl. I);

7) début d'une nouvelle séquence représentée par une surface d'érosion et les sédiments holocènes du Saint-Laurent.

\section{CONCLUSION}

L'inventaire des unités lithostratigraphiques et biostratigraphiques du Quaternaire de la vallée du Saint-Laurent n'est pas complètement terminé. Il existe notamment des unités encore peu connues sous le niveau du fleuve. La colonne lithostratigraphique actuellement connue peut être subdivisée en séquences sédimentaires. Les séquences sont caractéristiques d'une vallée tectoniquement stable, périodiquement englacée, soumise à des mouvements glacio-isostatiques et inondée. Les sédiments fluviatiles interglaciaires et interstadiaires sont emboîtés dans les séquences antérieures ou leurs témoins. 


\section{REMERCIEMENTS}

Les recherches ont été subventionnées par le programme de Formation des chercheurs et d'aide à la recherche du ministère de l'Éducation du Québec (équipe FCAR de C. HillaireMarcel), par le programme de développement du Réseau de I'Université du Québec (équipe Lancery et Occhietti) et par le Conseil de recherche en sciences naturelles et génie du Canada (subvention A7408). Ce texte émane des travaux de recherche menés en équipe avec $F$. Bernier, $F$. Besré et $P$. Ferland, de l'Université du Québec à Montréal, M. Clet, du Centre CNRS de géomorphologie de Caen, France, et J.-M. Lancery, de l'Université du Québec à Trois-Rivières. M. Parent, de la Commission géologique du Canada, à Québec, et P.J.H. Richard, de I'Université de Montréal, ont également collaboré à ces travaux. L'hospitalité, envers toute l'équipe, de Jocelyn Pelletier, de I'Université du Québec à Trois-Rivières, et de Claudie Segret et Jean Pelletier, de Sainte-Foy, a représenté l'équivalent d'une subvention. J.-S. Vincent et D. St-Onge, de la Commission géologique du Canada à Ottawa, ont révisé très attentivement ce texte.

\section{RÉFÉRENCES}

Anderson, T. W., Matthews, J. V. Jr., Mott, R. J. et Richard, S. H., 1988. The Pointe-Fortune interglacial site. In C. R. Harington, édit., Climatic Fluctuations and Man 3. Canadian Committee on climatic fluctuations and Man. Annual Meeting, Ottawa. Abstracts, 12-13.

Baulig, H., 1956. Vocabulaire franco-anglo-allemand de géomorphologie. Publications de la Faculté des lettres de l'Université de Strasbourg, Fascicule 130, 230 p.

Bernier, F. et Occhietti, S., 1990. Le complexe glaciaire du Cap Charles, vallée moyenne du Saint-Laurent, Québec. Géographie physique et Quaternaire, 44 (2) : 173-180

Besré, F. et Occhietti, S., 1990. Les Varves de Deschaillons, les Rythmites du Saint-Maurice et les rythmites de Leclercville, Pléistocène supérieur, vallée du Saint-Laurent, Québec. Géographie physique et Quaternaire, 44 (2): 181-198

Bhiry, N., Rognon, P. et Occhietti, S., sous presse. Origine et diagenèse des sédiments quaternaires de la plaine moyenne du Souss, Maroc. Sciences géologiques.

Brodeur, D. et Allard, M., 1985. Stratigraphie et Quaternaire à l'île aux Coudres, estuaire moyen du Saint-Laurent, Québec. Géographie physique et Quaternaire, 39 (2) : 183-197.

Clark, P. et Karrow, P. F., 1983. Till stratigraphy in the St. Lawrence Valley near Malone, New York. Revised glacial history and stratigraphy nomenclature. Geological Society of America. 94: 1306-1318.

Clet, M. et Occhietti, S., 1988. Palynologie des sédiments attribués à l'intervalle non-glaciaire de St-Pierre (Québec, Canada). Étude préliminaire. Actes $10^{\circ}$ Symposium de l'Association des palynologues de langue française. Institut français de Pondichéry, Travaux de la section scientifique et technique, XXV: 185-196.

Clet, M., Occhietti, S. et Richard, P. J. H., 1986. Stratigraphie et palynologie du Wisconsinien de la coupe de Donnacona, Québec. Annales de l'ACFAS, 54:216.

Dreimanis, A. et Karrow, P. F., 1972. Glacial history of Great Lakes St. Lawrence Region, the classification of the Wisconsin(an) Stage, and its correlatives. $24^{\mathrm{e}}$ Congrès international de géologie. Section 12: 5-15.
Elson, J. A., 1969. Radiocarbon dates, Mya arenaria phase of the Champlain Sea. Canadian Journal of Earth Sciences, 6 (3): 367-372.

Ferland, P. et Occhietti, S., 1990a. L'Argile de la Pérade: nouvelle unité marine antérieure au Wisconsinien supérieur, vallée du SaintLaurent, Québec. Géographie physique et Quaternaire, 44 (2): 159-172

1990b. Révision du stratotype des Sédiments de Saint-Pierre et implications stratigraphiques, vallée du Saint-Laurent, Québec. Géographie physique et Quaternaire, 44 (2): 147-158

Gadd, N. R., 1955. Pleistocene geology of the Bécancour map-area, Québec. Thèse de Ph.D., University of Illinois, Urbana, 191 p.

- 1960. Surficial geology of the Bécancour Map area, Québec. Geological Survey of Canada, Paper 59-8, 33 p.

- 1971. Pleistocene geology of the central St. Lawrence Lowland, with selected passages from an unpublished manuscript: The St. Lawrence Lowland, by J. W. Goldthwait. Geological Survey of Canada, Memoir 359, $153 p$.

Gadd, N. R., LaSalle, P., Dionne, J.-C., Shilts, W. W. et McDonald, B. C., 1972. Quaternary geology and geomorphology of southern Quebec. 24th International Congress of Geology, Montréal. Guide book, excursions A44-C44, $70 \mathrm{p}$.

Gadd, N. R., Richard, S. H. et Grant, D. R., 1981. Pre-last-glacial organic remains in Ottawa valley. In Current Research, Part C, Geological Survey of Canada, Paper 81-1C: 65-66.

Hillaire-Marcel, C. et Causse, C., 1989. Chronologie Th/U des concrétions calcaires des varves du lac glaciaire de Deschaillons (Wisconsinien inférieur). Canadian Journal of Earth Sciences, 26 (5): 1041-1052.

Karrow, P. F., 1957. Pleistocene geology of the Grondines map-area, Quebec. Thèse de Ph.D., University of Illinois, Urbana, $97 \mathrm{p}$.

- 1984. Quaternary stratigraphy and history, Great Lakes-St. Lawrence region, p. 137-153. In R. J. Fulton, édit., Quaternary Stratigraphy of Canada - A Canadian Contribution to IGCP Project 24. Geological Survey of Canada, 84-10.

Lamothe, M., 1985. Lithostratigraphy and geochronology of the Quaternary deposits of the Pierreville and St-Pierre-les-Becquets areas, Québec. Thèse de Ph.D., University of Western Ontario, London, $227 \mathrm{p}$.

1987. Pleistocene stratigraphy in the St. Lawrence Lowland, p. 15-41. In M. Lamothe, édit., Pleistocene stratigraphy in the St. Lawrence Lowland and the Appalachians of southern Québec: a field guide. Université de Montréal, Collection Environnement et géologie, 4, $126 \mathrm{p}$.

1989. A new framework for the Pleistocene stratigraphy of the central St. Lawrence Lowland, southern Québec. Géographie physique et Quaternaire, 43 (2): 119-129.

Lamothe, M. et Huntley, D. J., 1988. Thermoluminescence dating of late Pleistocene sediments, St. Lawrence Lowland, Québec. Géographie physique et Quaternaire, 42 (1) : 33-44.

LaSalle, P., 1984. Quaternary stratigraphy of Québec: A review. p. 155-171. In R. J. Fulton, édit. Quaternary Stratigraphy of Canada - A Canadian Contribution to IGCP Project 24. Geological Survey of Canada, Paper 84-10.

Lavoie, J., 1987. Étude morpho-sédimentologique du rivage de Grondines. Thèse de maîtrise, Département de géographie, Université Laval, Québec, 158 p.

Lécolle, F., 1984. Phases érosives et cycles sédimentaires: les alluvions de la Seine au sud du Vexin. Contexte paléoclimatique. 
Bulletin de l'Association française pour l'étude du Quaternaire, 1-2-3: 33-36.

MacClintock, P. et Stewart, D. P., 1965. Pleistocene geology of the St. Lawrence Lowland. New York State Museum and Science Service, Bulletin 394, 152p.

McDonald, B. C., 1971. Late Quaternary stratigraphy and deglaciation in eastern Canada, p. 331-353. In K. K. Turekian, édit., The late Cenozoic glacial ages. Yale University Press.

MacDonald, B. C. et Shilts, W. W., 1971. Quaternary stratigraphy and events in southeastern Canada. Geological Society of America Bulletin, 82: 683-698.

Miller, G. H., Andrews, J. T. et Short S. K, 1977. The last interglacialglacial cycle, Clyde foreland, Baffin Island, N.W.T.: stratigraphy, biostratigraphy, and chronology. Canadian Journal of Earth Sciences, 14 (12) : 2824-2857.

Occhietti, S., 1977. Stratigraphie du Wisconsinien de la région de TroisRivières Shawinigan, Québec. Géographie physique et Quaternaire, 31 (3-4): 307-322.

1982. Synthèse lithostratigraphique et paléoenvironnements du Quaternaire au Québec méridional. Hypothèse d'un centre d'englacement wisconsinien au Nouveau-Québec. Géographie physique et Quaternaire, 36 (1-2): 15-49.

Occhietti, S., Clet, M., Bernier, F., Besré, F., Ferland, P. et Lancery, J.-M., 1989. Contribution à la lithostratigraphie de la vallée du SaintLaurent: Saint-Pierre-les-Becquets, Sainte-Anne-de-la-Pérade et Donnacona, p. 35-53. In M. Lamothe, édit., Stratigraphie du Pléistocène du Québec méridional, Livret-Guide d'excursion A 1 , Réunion annuelle de l'Association des géologues du Canada, $118 \mathrm{p}$.

Occhietti, S. et Rutter, N., 1982. Acides aminés et interstades du Wisconsinien de la vallée du Saint-Laurent et de lî̀le du Cap Breton. In Recherches en cours, Partie B. Commission géologique du Canada, Étude 82-1B: 301-305.
Parent, M., 1987. Late Pleistocene stratigraphy and events of the Asbestos-Valcourt region, Southeastern Quebec. Thèse de Ph.D., University of Western Ontario, London, 320 p.

Parent, M. et Occhietti S., 1988. Late Wisconsinan ice retreat and Champlain Sea invasion in the St. Lawrence Valley, Québec. Géographie physique et Quaternaire, 42 (3) : 215-246.

Prest, V. K., 1975. Géologie du Quaternaire au Canada. Géologie et ressources minérales du Canada, partie $B$, chap. 12, 751-857.

Prest, V. K. et Hode-Keyser, J., 1977. Geology and engineering characteristics of surficial deposits, Montreal island and vicinity, Québec. Geological Survey of Canada, Paper 75-27, 29 p.

Prichonnet, G., 1982. Quelques données nouvelles sur les dépôts quaternaires du Wisconsinien et de l'Holocène dans le piedmont appalachien, Granby, Québec. In Recherches en cours, Partie B. Commission géologique du Canada, Étude 82-1B 225-238.

Sharpe, D. R. et Barnett, P. J., 1985. Significance of sedimentological studies on the Wisconsinan stratigraphy of southern Ontario. Géographie physique et Quaternaire, 39 (3) : 255-273.

Simard, L., 1971. Relevé des caractéristiques des roches et dépôts meubles de la vallée du Saint-Laurent. Ministère des Travaux publics, Division du chenal maritime du Saint-Laurent, Montréal, $1,331 \mathrm{p}$

Skinner, R. G., 1973. Quaternary stratigraphy of the Moose River Basin, Ontario. Geological Survey of Canada, Bulletin 46, 35 p.

Stuiver, M., Heusser, C. J. et Yang, I. C., 1978. North American glacial history extended to 75,000 years ago. Science, 200: 16-21.

Terasmae, J., 1958. Contributions to Canadian palynology, part 2; nonglacial deposits in the St-Lawrence Lowlands, Québec. Geological Survey of Canada, 46: 13-28.

Veillette, J. J. et Nixon, F. M., 1984. Sequence of the Quaternary Sediments in the Bélanger sand pit, Pointe-Fortune, QuébecOntario. Géographie physique et Quaternaire, 38 (1): 59-68.

Vincent, J.-S., 1983. La géologie du Quaternaire et la géomorphologie de l'île Banks, Arctique canadien. Commission géologique du Canada, Mémoire 405, 118 p. 